\title{
EYE TRACKING EM INTERFACE DO GOOGLE: A INFLUÊNCIA DO ELEMENTO "RICH SNIPPET"
}

\author{
EYE TRACKING EN INTERFAZ DE GOOGLE: LA \\ INFLUENCIA DEL ELEMENTO "RICH SNIPPET"
}

\author{
Cecilio Merlotti Rodas* \\ Silvana Aparecida Borsetti Gregório Vidotti**
}

\begin{abstract}
RESUMO:
Introdução: Na era de intensa produção de informações na qual se vive, as interfaces humano-computador podem ter um impacto considerável sobre o sucesso de um produto. É por meio das interfaces que as pessoas acessam as informações e nesse contexto o usuário sempre deve ser considerado. Tendo em vista que o acesso às Tecnologias de Informação e Comunicação tem sido feito cada vez mais por pessoas menos especialistas, como crianças e idosos, as interfaces precisam se tornar gradativamente mais intuitivas e serem capazes de atrair a atenção de seus usuários. Nesse contexto, estudos em User Experience mostram que certas tecnologias, como o Eye Tracking, são capazes de capturar camadas extras de dados e assim permitir uma melhor compreensão do comportamento das pessoas ao acessarem informações digitais. Objetivo: Nos testes buscou-se identificar se os usuários poderiam ser influenciados pelas duas diferentes tarefas dadas, uma com e outra sem apelo emocional. Metodologia: Neste trabalho foram realizados testes com usuários sobre a página de resultados do mecanismo de busca do Google, utilizando a tecnologia de Eye Tracking, procurando estimular o apelo emocional em um dos dois grupos participantes. Resultados: Os resultados surpreenderam ao mostrar que os links contidos nas páginas de resultados do Google, que apresentavam Rich Snippets, foram capazes de influenciar o comportamento dos participantes, independente da tarefa. Conclusão: Esses resultados revelaram o impacto do elemento Rich Snippet na experiência do usuário ao buscar informações na interface do Google.
\end{abstract}

\footnotetext{
* Doutor em Ciência da Informação pela Universidade Estadual Paulista Júlio de Mesquita Filho (Unesp). Professor do Instituto Federal de Educação Ciência e Tecnologia de São Paulo (IFSP) Campus Votuporanga. E-mail: cecilio.rodas@gmail.com

${ }^{* *}$ Doutora em Educação pela Universidade Estadual Paulista Júlio de Mesquita Filho (Unesp). Professora do Programa de Pós-Graduação em Ciência da Informação da Universidade Estadual Paulista Júlio de Mesquita Filho (Unesp) Campus de Marília. E-mail: vidotti@reitoria.unesp.br.
}

Inf. Inf., Londrina, v. 22, n. 2, p. 389 - 419, maio/ago., 2017. 
Palavras-chave: Eye tracking. Google. Páginas de resultados de mecanismo de busca. Rich Snippet. Interação homem-computador.

\title{
1 INTRODUÇÃO
}

$\mathrm{Na}$ era de intensa produção de informações na qual se vive, as interfaces humano-computador podem ter um impacto considerável sobre 0 sucesso de um produto já que é a partir delas que o usuário poderá ser capaz de perceber o quanto elas podem colaborar, por exemplo, no processo de recuperação de informações (JOHNSON, 2010).

Sabendo que as emoções podem influenciar nas escolhas de informação das pessoas e sabendo que nem sempre tais emoções podem ser percebidas sem a utilização de aparatos tecnológicos, algumas tecnologias estão sendo utilizadas em testes com usuários com a finalidade de melhorar a qualidade dos resultados de testes em User Experience (UX), inclusive em estudos de interfaces de ambientes informacionais digitais (BERGSTROM; SCHALL, 2014).

Segundo Bergstrom e Schall (2014), em alguns casos, a pesquisa em UX limita-se a comportamentos observáveis e isso pode fazer com que nem todos os dados sejam coletados adequadamente:

\begin{abstract}
Através da utilização de entrevistas, questionários ou protocolo de verbalização, os pesquisadores devem confiar na memória de um participante e de seus julgamentos subjetivos como um meio de obter insights sobre seus próprios processos cognitivos e estados emocionais. A neurociência cognitiva entende que a percepção subjetiva de uma pessoa sobre o seu próprio comportamento nem sempre corresponde à sua atividade neuronal subjacente. Ou seja, as pessoas nem sempre têm plena consciência do que está acontecendo dentro suas próprias mentes. (BERGSTROM; SCHALL, 2014, p.89, tradução nossa)
\end{abstract}

Tendo em vista esta observação é possível que uma pessoa possa, sem perceber, omitir informações relevantes durante um teste, sem que se dê conta. Assim, a adição de novas tecnologias em testes com usuários tem a 
finalidade de adicionar novas camadas de dados que permitam encontrar melhores resultados em pesquisas de UX.

Assim, Bergstrom e Schall (2014) mostram a importância de se adicionar ferramentas que sejam capazes de recuperar dados que vão além da avaliação subjetiva de uma pessoa. De acordo com Bergstrom e Schall (2014), um exemplo dessas tecnologias é capaz de medir sensíveis alterações da secreção de suor na pele da mão de um participante durante um teste de UX por meio de um sensor de resposta galvânica da pele (galvanic skin response GSR). Acredita-se que a alteração repentina de secreção de suor na pele de uma pessoa esteja relacionada a fortes emoções. De tal modo, durante um teste de uma interface, por exemplo, tais alterações poderiam significar que um ou mais elementos foram capazes de gerar sentimentos e/ou emoções e consequentemente impressionar o usuário.

Outra tecnologia que tem sido incorporada em pesquisas com usuários é conhecida como Eye Tracking (BERGSTROM; SCHALL, 2014). Essa tecnologia grava o olhar do usuário sobre um determinado elemento visual, assim, é possível obter dados mais precisos sobre a percepção e experiência dos usuários ao visualizar determinados estímulos. A pertinência de estudos envolvendo Eye Tracking tem como base a hipótese de que aquilo que uma pessoa visualiza é assumido como indicador do pensamento atual/dominante nos processos cognitivos (BARRETO, 2012).

Entendendo que as emoções podem balizar o comportamento dos indivíduos ao visualizarem elementos visuais, neste trabalho procurou-se verificar por meio de testes com usuários se o apelo emocional de uma dada tarefa poderia influenciar o comportamento dos usuários participantes.

Assim foi desenvolvido dois testes com humanos, utilizando-se a tecnologia de Eye Tracking para investigar se o comportamento do olhar dos usuários poderia ser afetado, durante a busca de informação, ao se utilizar uma palavra que pudesse despertar sentimentos e/ou emoções na descrição da tarefa a ser executada. 
O teste foi aplicado sobre a interface do mecanismo de busca do Google, avaliando o impacto do elemento visual conhecido como Rich Snippet. Por ser um elemento visualmente enriquecido, contendo informações a partir de dados estruturados, ele permite apresentar as informações não somente em forma textual, mas também com imagens ilustrativas, estrelas de avaliação, entre outros. Há relatos que esses novos elementos inseridos nas páginas de resultados do Google têm provocado a alteração do comportamento de seus usuários (MAYNES; EVERDELL, 2014).

Rich Snippet é uma pequena descrição que aparece logo abaixo do link de alguns resultados apresentados nas páginas dos mecanismos de busca, como o Google, e é criado automaticamente com base no conteúdo do site ou das páginas vinculadas a ele. A palavra "rich", segundo Google (2016), se refere à nova característica desse elemento que permite sua composição a partir de dados estruturados, o que possibilita uma melhor indexação pelos mecanismos de busca, além da possibilidade de se criar uma visualização mais 'rica' das informações nele contidas.

Assim, buscou-se identificar se os Rich Snippet seriam capazes de influenciar sobremaneira o olhar dos usuários que tivessem uma tarefa com apelo emocional. Ou se, sendo eles enriquecidos visualmente, seriam capazes de influenciar em ambas tarefas, com ou sem apelo emocional, o que evidenciaria a importante influência de tais elementos.

\section{EYE TRACKING EM USER EXPERIENCE}

O User Experience é um termo que nomeia os estudos que visam melhorar a experiência do usuário no contexto da interação humanocomputador (IHC), aperfeiçoando a usabilidade dos sistemas (BERGSTROM; SCHALL, 2014).

De acordo Klein e Bulla (2010), os estudos que envolvem a tecnologia de Eye Tracking estão interessados em verificar a atividade dos olhos diante de determinados estímulos visuais. Os movimentos oculares importantes registrados pela tecnologia de Eye Tracking são conhecidos como fixações e 
sacadas (KLEIN; BULLA, 2010; BOJKO, 2013). As fixações são os períodos de tempo durante os quais os olhos permanecem parados examinando uma pequena área do estímulo, podendo durar de 100 a 500 ms (milissegundos); e as sacadas são pequenos movimentos realizados entre as fixações, podendo durar de 10 a 100 ms (KLEIN; BULLA, 2010; PERNICE; NIELSEN, 2009), como pode ser visto na figura 1 .

Um estímulo em um estudo de UX, segundo Rodas, Marcos e Vidotti (2014, p. 3) "[...] pode ser uma interface (uma página Web, uma imagem) ou um objeto em um local aberto (uma prateleira, uma embalagem)." Ou seja, dentro deste contexto, um estímulo pode ser qualquer elemento visual capaz de ser recuperado pela tecnologia de Eye Tracking.

Figura 1 - Exemplo de fixações (círculos) e as sacadas (linhas entre os círculos)

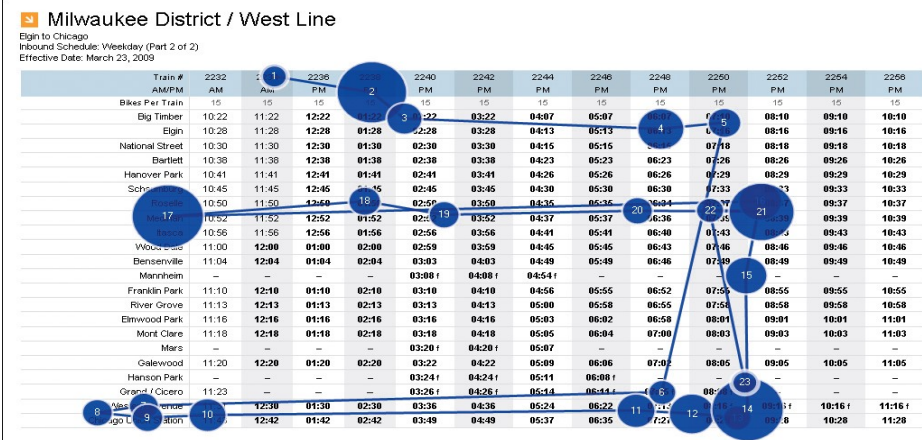

Fonte: BOJKO (2013, p.12)

Para Barreto (2012), a pertinência de estudos envolvendo Eye Tracking tem como base a hipótese de que aquilo que uma pessoa visualiza é assumido como indicador do pensamento atual/dominante nos processos cognitivos. Diante de uma interface, a ação, a aceitação, a compreensão ou o reconhecimento dos elementos informacionais pelo usuário pode ter razões diferentes, dependendo ou não do que foi visualizado durante os testes (BOJKO, 2013).

A Tecnologia de Eye Tracking permite encontrar resultados mais precisos, capazes de mostrar o que ocorre, por exemplo, entre os "cliques" do mouse. Com o uso de Eye Tracking é possível compreender porque um 
usuário não completou uma tarefa, mostrando se ele deveria "clicar" ${ }^{1}$ em um determinado link e não o fez. O Eye Tracking também pode oferecer informações sobre a eficácia, ou seja, o esforço necessário para concluir uma tarefa com êxito. Ainda permite mostrar como uma interface pode influenciar no comportamento das pessoas, adicionando informações que poderiam não ser percebidas com outros tipos de testes de UX, como o protocolo de verbalização (thinking aloud protocol), por exemplo (BERGSTROM; SHAWN, 2014).

Os principais tipos de visualizações oferecidas pelos softwares que acompanham a tecnologia de Eye Tracking podem ser vistos na figura 2. São elas, os mapas estáticos de calor (heat map) (figura 2-a); mapa estáticos de opacidade (opacity gaze map) (figura 2-b); mapa estático do olhar (Gaze Plot ou ScanPaths) (figura 2-c). Tais mapas são capazes de fornecer uma primeira visão geral por meio de imagens estáticas geradas pela sobreposição dos mapas individuais de cada usuário. É possível gerar também vídeos do olhar mostrando as fixações e sacadas em modo dinâmico para um ou mais usuários. (RODAS; MARCOS; VIDOTTI, 2014).

Figura 2 - Visualizações mais comuns para resultados de UX utilizando Tecnologia de Eye Tracking: (a) mapa de calor (heat map); (b) mapa de opacidade (opacity gaze map); (c) mapa do olhar (gaze plot)
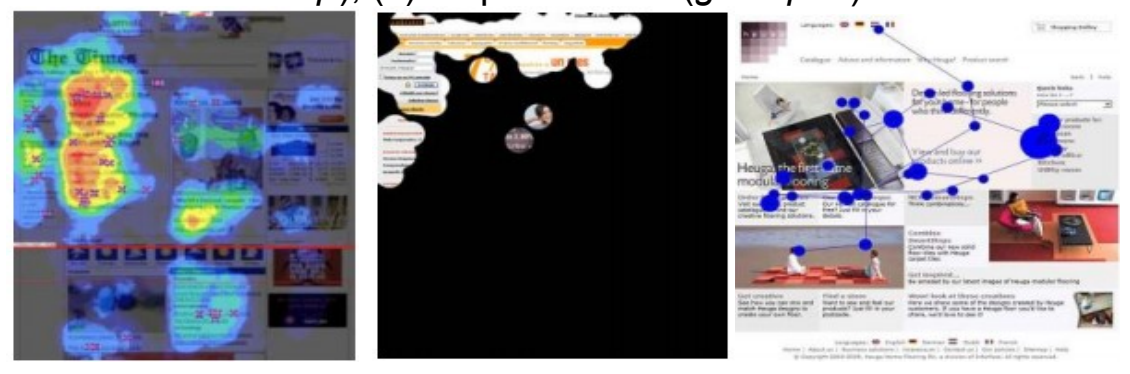

Fonte: Barreto (2012, p. 178 e 179)

Como visto anteriormente, em testes tradicionais de UX é possível que uma pessoa possa, sem perceber, omitir informações relevantes durante um

\footnotetext{
1 O termo "clicar" neste trabalho representa a ação do usuário de pressionar com o dedo o botão do mouse. Essa ação também é registrada pelo sistema de Eye Tracking e representa a escolha dos usuários participantes dos testes.
}

Inf. Inf., Londrina, v. 22, n. 2, p. 402 - 419, maio/ago., 2017. http:www.uel.br/revistas/informacao/ 
teste, sem que se dê conta. Assim, a adição de novas tecnologias em testes com usuários tem a finalidade de adicionar novas camadas de dados que permitam encontrar melhores resultados em pesquisas de UX.

Portanto, considerando que a tecnologia de Eye Tracking oferece considerável precisão em testes com UX, considerando ainda a importância de se adicionar tais camadas de dados aos estudos relacionados com a experiência de usuários diante de interfaces em telas de computadores, pretende-se justificar o seu uso nos testes realizados neste trabalho.

\section{METODOLOGIA}

Para o desenvolvimento deste trabalho seguiu-se um método de investigação experimental por meio da realização de testes com usuários utilizando a Tecnologia de Eye Tracking para recuperar dados referentes ao olhar dos usuários sobre a interface da primeira página de resultados do Google ao buscar uma determinada informação. O objetivo foi verificar se uma palavra que desse uma conotação de "sentimento" ou de "emoção" poderia balizar o comportamento do usuário ao buscar uma informação diante de resultados contendo Rich Snippet. Assim, procurou-se verificar como o usuário se comportaria ao buscar uma informação para uma "receita de bolo de cenoura", porém com tarefas ligeiramente diferentes. Nesse sentido foram realizados dois testes, com dois grupos diferentes de pessoas.

$\mathrm{Na}$ tarefa de um dos grupos foi solicitado que o usuário encontrasse, diante dos resultados apresentados na SERP (search engine results page página de resultados de mecanismos de busca) do Google, um resultado que Ihe desse condições para "saber como fazer um bolo de cenoura". Na tarefa do segundo grupo foi acrescentada uma palavra "gostoso", assim os participantes deveriam procurar um resultado que permitisse encontrar uma receita para "saber como fazer um 'gostoso' bolo de cenoura".

Nesse sentido procurou-se identificar se a palavra "gostoso" teria um impacto no comportamento do usuário, fazendo com que os sentimentos pudessem influenciar nas escolhas das pessoas, tendo em vista que a palavra 
"gostoso" poderia influenciar os participantes a buscarem, na interface, por resultados que pudessem trazer outros tipos de informações, como imagens de alimentos apetitosos, além de texto simplesmente.

\subsection{Procedimentos Metodológicos}

Antes de cada teste apresentava-se a Tecnologia de Eye Tracking assim como o Eye Tracker (dispositivo capaz de recuperar e gravar o olhar do usuário) para que o usuário pudesse se sentir familiarizado com os procedimentos a serem realizados. Em seguida era feita a calibragem para cada um dos participantes para que o Eye Tracker fosse capaz de recuperar e gravar os dados referentes ao olhar do usuário sobre o estímulo.

No início do teste o usuário tinha que ler a tarefa a ser realizada na própria tela do computador e quando tivesse terminado de ler deveria "clicar" em qualquer lugar na tela de instruções para que o teste fosse iniciado. Diante do estímulo apresentado, a SERP do Google, o participante tinha a tarefa de procurar por um resultado que apresentasse uma receita para "fazer um bolo de cenoura", ou, no segundo caso, uma receita para "fazer um 'gostoso' bolo de cenoura". Os participantes foram instruídos para que "clicassem" sobre o link escolhido assim que tivessem tomado uma decisão.

Após o "clique" sobre o resultado escolhido o usuário deveria fechar o navegador, momento no qual o teste se encerrava com uma tela contendo uma mensagem de agradecimento. Em seguida o participante era convidado a responder a um questionário sobre seus hábitos de busca na Web.

Os usuários participaram de forma voluntária, o consentimento se deu via documento conhecido como Termo de Consentimento Livre Esclarecido (TCLE) autorizando a utilização dos dados coletados para fins acadêmicos. A pesquisa foi autorizada pelo Comitê de Ética em Pesquisa da Faculdade de Filosofia e Ciências da Unesp - Campus de Marília / Plataforma Brasil, identificada pelo registro CAAE: 54877216.8.0000.5406. 


\subsection{Apresentação do Estímulo}

Em testes com Eye Tracking, estímulo é o nome que se dá ao objeto de estudo, podendo ser qualquer tipo de elemento visual desde que haja a possibilidade de ser rastreado por meio de um Eye Tracker, como as imagens em uma tela de um computador, por exemplo. Assim, o estímulo (figura 3) foi elaborado a partir da imagem da primeira página resultados do Google (2015), e apresentado em um navegador (browser) dentro de um ambiente controlado que permitia ao usuário ter a sensação de estar online. Tal estímulo foi apresentado em uma tela LCD de 19 polegadas com resolução de $1024 \times 768$. Os testes deste trabalho foram projetados com o software Tobii Studio, versão 3.4.0, utilizando o Eye Tracker modelo Tobii X2-60.

Figura 3 - Imagem contendo a primeira página de resultados para uma busca realizada no Google com as palavras-chave "receita de bolo de cenoura"

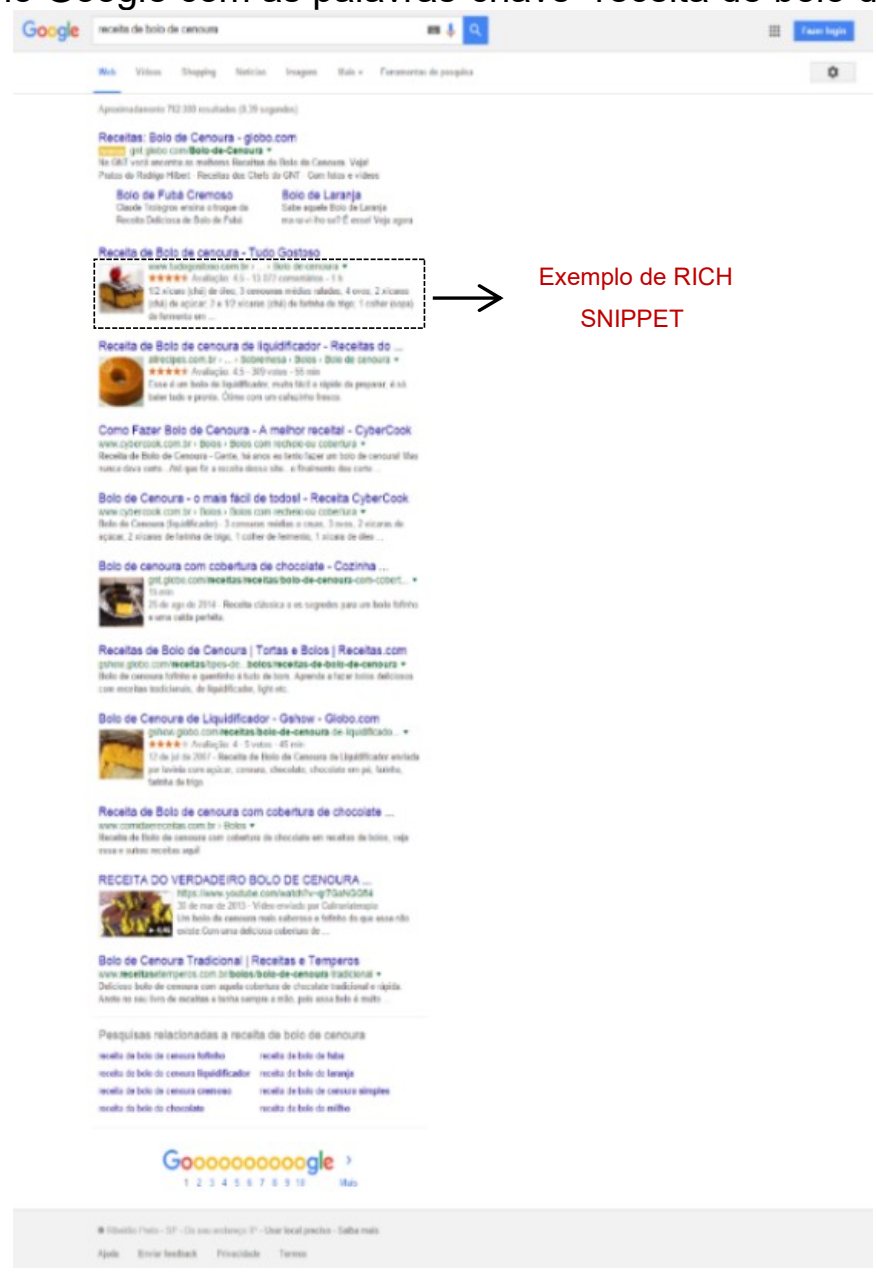

Fonte: Elaborado pelos autores

Inf. Inf., Londrina, v. 22, n. 2, p. 402 - 419, maio/ago., 2017. http:www.uel.br/revistas/informacao/ 


\subsection{A Amostra}

Para a realização do teste foram selecionados aleatoriamente vinte estudantes de graduação de cursos da Unesp na cidade de Marília, local onde o experimento ocorreu. Os participantes se dividiram em dois grupos de dez pessoas cada. Segundo Nielsen (2000), testes com cinco participantes permitem identificar aproximadamente cerca de $85 \%$ de problemas relacionados à usabilidade, sendo assim, considera-se que a quantidade de participantes para este estudo tenha sido satisfatória.

Dentre as pessoas que participaram, a proporção de homens foi de $35 \%$ e $65 \%$ de mulheres, com idade média de 25 anos. Os participantes relataram que fazem uso da Internet em média há dez anos. Nenhum dos participantes relatou ter participado de testes com a tecnologia de Eye Tracking anteriormente. Os usuários também não tinham conhecimento prévio das tarefas a serem realizadas durante os testes, evitando assim que sofressem qualquer tipo de influência.

\section{RESULTADOS}

Entre algumas das características dos participantes destacamos que, 95\% afirmaram utilizar o Google como principal buscador devido principalmente à qualidade dos resultados, ao layout de sua interface e também por sua popularidade. Cerca de $70 \%$ dos entrevistados utilizavam o site do Google como página principal em seus navegadores (browsers), e é a partir dele que iniciavam seus acessos à Web.

Para a análise dos resultados foram criadas áreas de interesse (AOl's) para identificar os resultados com Rich Snippet destacado com a cor verde na figura 4, e os que não continham Rich Snippet (neste caso, continham apenas snippet, ou seja, os snippet tradicionais, contendo apenas uma descrição textual) com a cor vermelha na mesma figura.

Dois grupos de usuários fizeram a mesma busca, diante de uma mesma interface de resultados no mecanismo de busca do Google. A palavra "gostoso", porém, foi retirada da tarefa que foi dada a um dos grupos, com a 
finalidade de verificar se o grupo que não tinha o apelo emocional pudesse ter seu comportamento alterado, fazendo com que buscassem por links contendo menos informações híbridas, ou seja, sem Rich Snippet.

Figura 4 - Demarcação das áreas de interesse mostrando resultados contendo Rich Snippet (cor verde), e resultados sem Rich Snippet (cor vermelha)

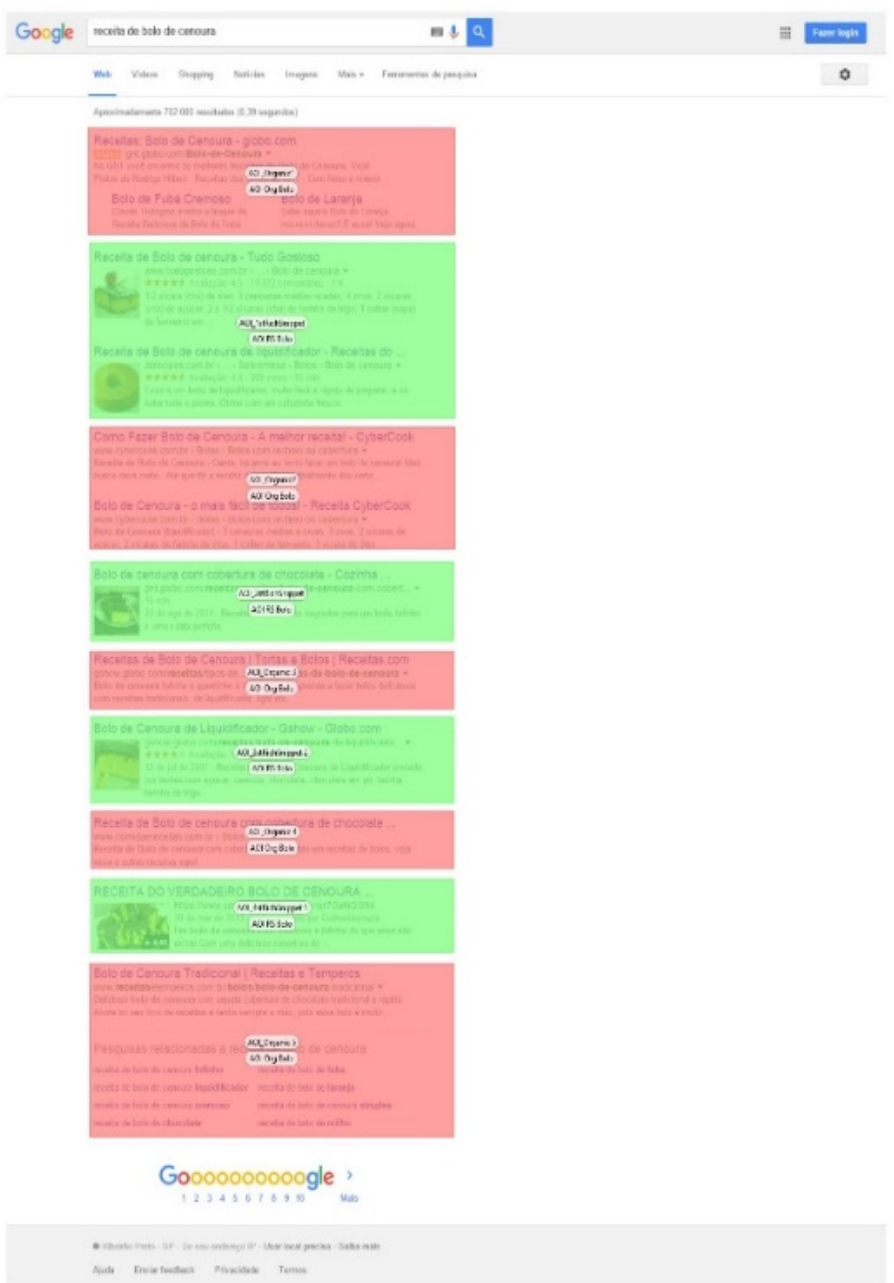

Fonte: Elaborado pelos autores

Os resultados mostraram, conforme gráfico 1 , que cerca de $68 \%$ do tempo de fixação dos olhares dos participantes foi sobre as AOl's que continham Rich Snippet (figura 4 - cor verde). O restante, cerca de $32 \%$ foi sobre as demais AOl's que não continham Rich Snippet (figura 4 - cor vermelha).

Inf. Inf., Londrina, v. 22, n. 2, p. 402 - 419, maio/ago., 2017. http:www.uel.br/revistas/informacao/ 
Gráfico 1 - Porcentagem do tempo em que os usuários fixaram seus olhares sobre as AOl's contendo Rich Snippet em comparação com as AOl's que continham apenas snippets

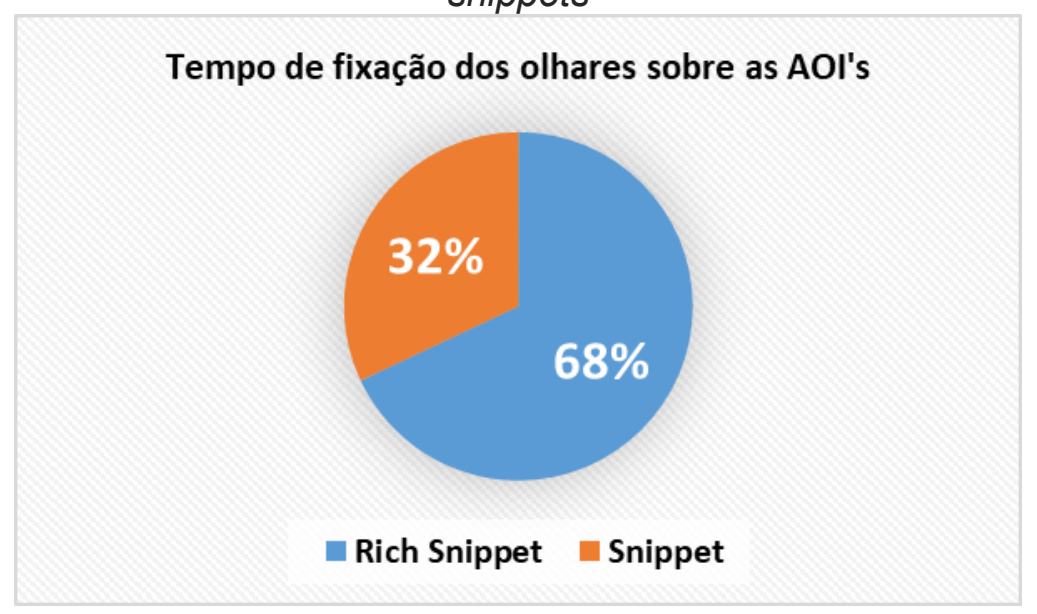

Fonte: Elaborado pelos autores

Esse resultado já é capaz de evidenciar como os links contendo Rich Snippet foram capazes de influenciar de modo muito mais significativo o olhar dos usuários do que os demais, já que os participantes passaram mais que o dobro do tempo visualizando esses links na SERP.

Na figura 5 é possível observar o mapa de calor gerado para o teste em que os usuários não tinham, na tarefa, o apelo emocional. Nela é possível observar que os olhares percorreram boa parte do estímulo e que a grande maioria dos participantes fixaram seus olhares em links que possuíam resultados contendo Rich Snippet. Além disso os dados mostram que 95\% do total de cliques ocorreram sobre os links que continham Rich Snippet.

A figura 6 mostra a visualização do mapa de calor para o teste realizado em que, na tarefa dada aos participantes, havia o apelo emocional, ou seja, a inserção da palavra "gostoso". Neste caso observa-se que os olhares dos usuários se concentraram mais nos primeiros links, principalmente naqueles que continham Rich Snippet. Os dados mostram que 100\% dos usuários "clicaram" sobre um único link e este possuía Rich Snippet.

Inf. Inf., Londrina, v. 22, n. 2, p. 402 - 419, maio/ago., 2017. http:www.uel.br/revistas/informacao/ 
Cecilio Merlotti Rodas, Silvana Aparecida Borsetti Gregório Vidotti

Eye tracking em interface do google: a influência do elemento "Rich Snippet"

Figura 5 - Visualização do mapa de calor para a busca realizada a partir de uma tarefa sem apelo emocional e/ou sentimental

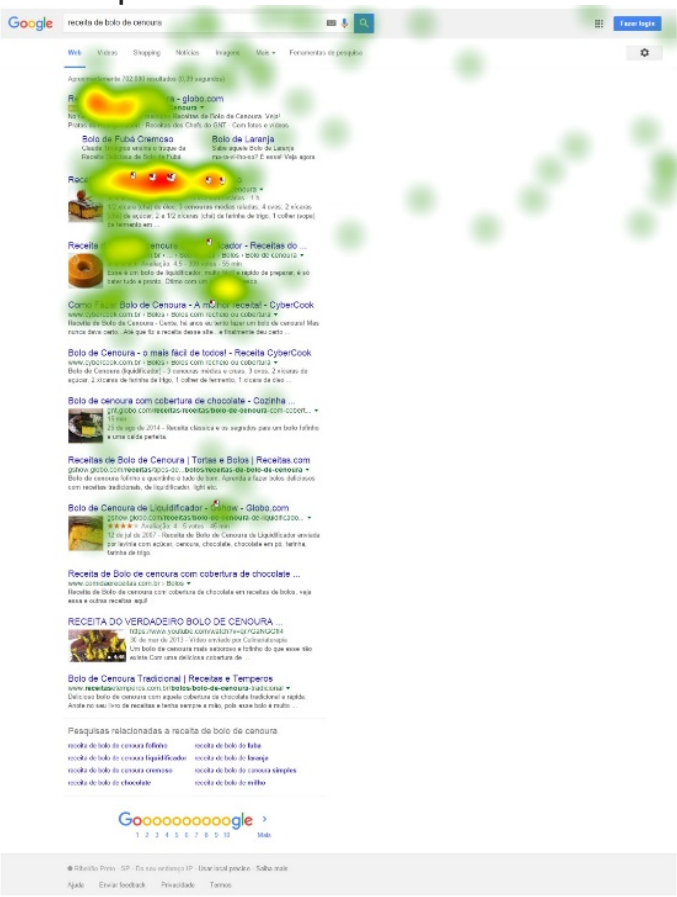

Fonte: Elaborado pelos autores

Figura 6 - Visualização do mapa de calor para a busca realizada a partir de uma tarefa com apelo emocional e/ou sentimental

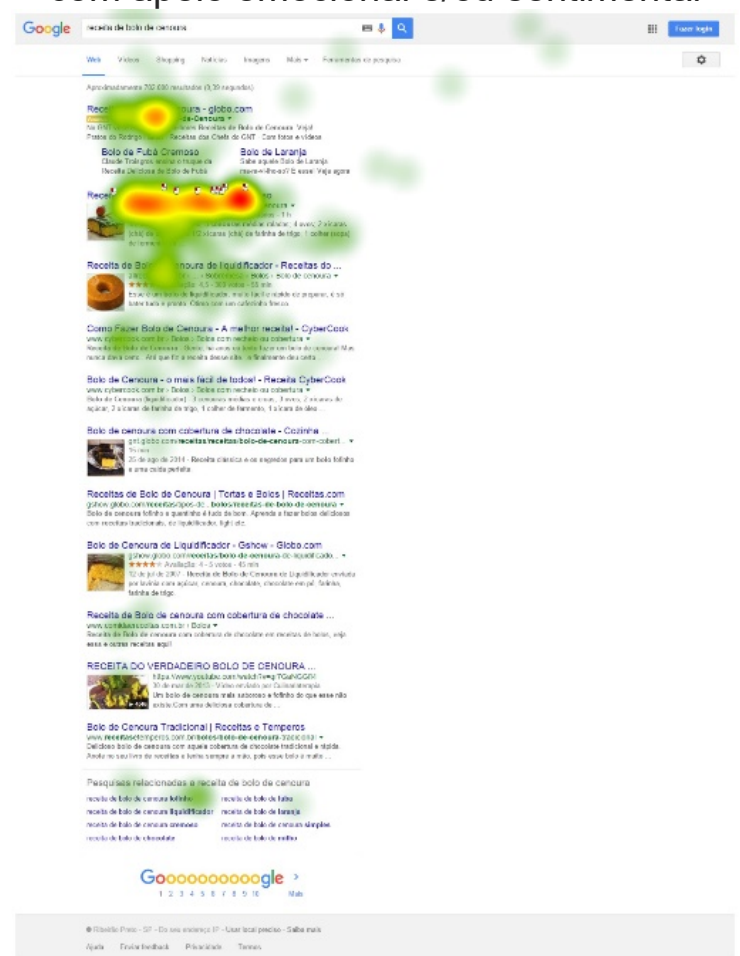

Fonte: Elaborado pelos autores

Inf. Inf., Londrina, v. 22, n. 2, p. 402 - 419, maio/ago., 2017. http:www.uel.br/revistas/informacao/ 
$\mathrm{Na}$ análise dos resultados foi possível observar que os resultados contendo Rich Snippet foram capazes de influenciar a escolha dos resultados nos dois grupos de usuários. Portanto, é possível inferir que, nas SERP, os resultados contendo Rich Snippet são fortes influenciadores independente da tarefa, com ou sem apelo emocional.

Pelos resultados deste trabalho também é possível concluir que as pessoas podem ter seu comportamento de busca influenciado para, na SERP do Google, os links contendo Rich Snippet. Isso mostra a capacidade que tais elementos com conteúdos híbridos, com imagens, textos, estrelas de avaliação, entre outros, podem ter sobre a decisão de escolha das pessoas ao acessarem as páginas de resultados do referido mecanismo de busca.

\section{CONSIDERAÇÕES FINAIS}

Neste trabalho teve-se o objetivo de identificar como seria 0 comportamento dos usuários diante de elementos da página de resultados do Google, conhecidos como Rich Snippet, estimulando-os por meio de dois tipos de tarefas, uma com apelo aos sentimentos e/ou emoções, e outra sem tal apelo.

A palavra "gostoso" inserida em uma das tarefas teve o intuito de gerar um apelo emocional e/ou sentimental nos usuários. Como eles estavam buscando por uma receita de bolo, imaginou-se que tal palavra, inserida na tarefa de um dos grupos, pudesse estimular a busca por resultados que mostrassem imagens, e outros tipos de informações, além das tradicionais informações textuais.

Como foi possível observar, $95 \%$ de todos os usuários, considerando os dois testes conjuntamente, preferiram "clicar" sobre um link que continha Rich Snippet. Além disso verificou-se que os links contendo Rich Snippet foram capazes de influenciar de modo muito mais significativo o olhar dos usuários, já que os dados mostraram que os participantes passaram mais que o dobro do 
tempo visualizando tais links na SERP.

No teste cuja tarefa tinha apelo emocional, $100 \%$ dos usuários escolheram um link que continha Rich Snippet, e no teste sem apelo emocional, 90\% escolheram links com Rich Snippet. Destaca-se o alto índice, de $90 \%$, do grupo de usuários que realizaram a tarefa sem apelo emocional, também terem escolhido links com Rich Snippet.

Esses números são relevantes, pois demonstram a capacidade que o Rich Snippet, contendo elementos informacionais, com texto, foto, estrelas classificatórias, pode ter sobre a decisão dos usuários ao buscar informação em páginas de mecanismos de busca. Apesar de se ter criado um teste tendo em vista que as emoções poderiam balizar os resultados, chegou-se à conclusão que os Rich Snippet foram capazes de alcançar índices consideráveis em ambos casos.

Assim, por meio dos resultados deste trabalho foi possível constatar que as informações híbridas contidas no elemento Rich Snippet da interface do Google pode estimular fortemente o olhar das pessoas. Tais constatações ressaltam ainda a importante consideração que as organizações devem dar às mudanças feitas nas interfaces dos mecanismos de busca, cientes de que isso poderá influenciar a experiência dos usuários ao buscar informações e, consequentemente, na visibilidade de seus ambientes informacionais digitais na Web.

\section{REFERÊNCIAS}

BARRETO, Ana Margarida. Eye Tracking como método de investigação aplicado às Ciências da Comunicação. Comunicando, v. 1, n. 1, p. 168-186, dez. 2012. Disponível em:

<http://www.revistacomunicando.sopcom.pt/edicao/12>. Acesso em 27 fev. 2014.

BERGSTROM, Jennifer Romano; SCHALL, Andrew Jonathan. Eye Tracking in User Experience Design. Waltham: Morgan Kaufmann, 2014. 
BOJKO, Aga. Eye tracking the user experience. New York: Rosenfeld Media, 2013.

GOOGLE. Rich Snippet. [S.I.]: Google, 2016. Disponível em:

$<$ https://developers.google.com/structured-data/rich-snippets>. Acesso em: 4 jan. 2016.

GOOGLE. Página de resultados do Google para a busca "receita de bolo de cenoura". [S.I.]: Google, 2015. Disponível em <www.google.com.br>. Acesso em: 5 nov. 2015.

JOHNSON, Jeff. Designing with the Mind in Mind Simple Guide to Understanding User Interface Design Rules. Burlington: Morgan Kaufmann Publishers, 2010.

KLEIN, Angela Inês; BULLA, Julieane Pohmann. Eye-Tracking e linguística: aplicações e interfaces. Letrônica: Revista Digital do PPGL, v. 3, n. 2, p. 235249, 2010. Disponível em:

$<$ http://revistaseletronicas.pucrs.br/ojs/index.php/\%20letronica/article/view/7606 >. Acesso em: 02 maio 2012.

MAYNES, Rebecca; EVERDELL, lan. L'évolution des pages de résultats de recherche Google et leurs effets sur le comportement des utilisateurs.

[S.I.]: Mediative, 2014. Disponível em: <http://www.mediative.com/fr/evolutiondes-pages-de-resultats-de-recherche-de-google-et-les-effets-sur-lesutilisateurs>. Acesso em: 17 jun. 2015.

NIELSEN, Jakob. Why You Only Need to Test with 5 Users. [S.I.]: NNGroup, 2015. Disponível em: <https://www.nngroup.com/articles/why-you-only-need-totest-with-5-users>. Acesso em: 9 nov. 2015.

PERNICE, Kara; NIELSEN, Jakob. How to conduct eyetracking studies. Fremont: Nielsen Norman Group, 2009. Disponível em:

$<$ http://www.nngroup.com/reports/how-to-conduct-eyetracking-studies>. Acesso em: 23 maio 2012.

RODAS, Cecilio Merlotti; MARCOS, Mari-Carmen; VIDOTTI, Silvana Aparecida Borsetti Gregório. Tecnologia de Eye Tracking em User Experience. In: ENCONTRO NACIONAL DE GESTÃO, POLITICAS E TECNOLOGIAS DE INFORMAÇÃO, 2014, Goiânia. Anais... Goiânia: UFG, 2014. p. 1-8.

Title

Eye Tracking in Google interface: the influence of the element "Richnippet"

Inf. Inf., Londrina, v. 22, n. 2, p. 402 - 419, maio/ago., 2017. http:www.uel.br/revistas/informacao/ 


\begin{abstract}
:
Introduction: In an era of intense production of information in which we live, the human-computer interfaces may have a considerable impact on the success of a product. It is through interfaces that people access information and in this context the user should always be considered. Considering that the access to Information and Communication Technologies has been frequently made by people less expert such as children and the elderly, interfaces need to become gradually more intuitive and able to attract the attention of its users. In this context, studies on User Experience shows that some technologies, such as Eye Tracking, are able to add extra layers of data and thus allow a better understanding of people's behavior when accessing digital information. Objective: In the tests we sought to identify whether users could be influenced by two different tasks, one with emotional appeal and another without emotional appeal. Methodology: This work carried out some tests about the access of the results page of the Google search engine using the Eye Tracking Technology, trying to stimulate the emotional appeal in one of the two participating groups. Results: The results were surprising and showed that the links in the Google results pages, with Rich Snippets, were able to influence the behavior of participants, regardless of the task. Conclusions: These results revealed the impact of Rich Snippet element in the users experience when they are seeking information on Google interface.
\end{abstract}

Keywords: Eye tracking. Google. Search engine result pages. Rich Snippet. Humancomputer interaction.

\title{
Titulo
}

Eye Tracking en interfaz de Google: la influencia del elemento "Rich Snippet"

RESUMEN: Introducción: En la era de intensa producción de informaciones en la cual se vive, las interfaces humano-computador pueden tener un impacto considerable sobre el éxito de un producto. Es por medio de las interfaces que las personas acceden a las informaciones y en este contexto el usuario siempre debe ser considerado. Considerando que el acceso a las Tecnologías de la Información y la Comunicación ha sido realizado cada vez más por personas menos expertas, como niños y ancianos, las interfaces necesitan tornarse gradualmente más intuitivas y ser capaces de atraer la atención de sus usuarios. En este contexto, estudios en Experiencia de Usuario demuestran que ciertas tecnologías, como Eye Tracking, son capaces de capturar niveles adicionales de datos y así permitir una mejor comprensión del comportamiento de las personas al acceder a informaciones digitales. Objetivo: En las pruebas se buscó identificar si los usuarios podrían ser influenciados por las dos tareas diferentes dadas, una con y otra sin atractivo emocional. Metodología: En este trabajo fueron realizadas pruebas con usuarios sobre la página de resultados del motor de búsqueda de Google, utilizando la tecnología de Eye Tracking, buscando estimular el atractivo emocional en uno de los dos grupos participantes. Resultados: Los resultados sorprendieron al mostrar que los enlaces contenidos en las páginas de resultados de Google, que presentaban Rich Snippets, fueron capaces de influir en el comportamiento de los participantes, independientemente de la tarea. Conclusiónes: Estos resultados revelaron el impacto del elemento Rich Snippet en la experiencia del usuario al buscar informaciones en la interfaz de Google.

Inf. Inf., Londrina, v. 22, n. 2, p. 402 - 419, maio/ago., 2017. http:www.uel.br/revistas/informacao/ 
Cecilio Merlotti Rodas, Silvana Aparecida Borsetti Gregório Vidotti

Eye tracking em interface do google: a influência do elemento "Rich Snippet"

Palabras clave: Eye Tracking. Google. Páginas de resultados de mecanismo de búsqueda. Rich Snippet. Interacción humano-computador

Recebido: 30.08.2017

Aceito: 30.09 .2017

Inf. Inf., Londrina, v. 22, n. 2, p. 402 - 419, maio/ago., 2017. http:www.uel.br/revistas/informacao/ 